\title{
El farmacéutico comunitario, necesario en el abordaje de la hipertensión arterial y el riesgo vascular
}

\author{
Montserrat Iracheta, ${ }^{1}$ Ana Molinero ${ }^{2}$ \\ 1. Vocal de la Junta Directiva de SEFAC. 2. Vicepresidenta de la Junta Directiva de SEFAC.
}

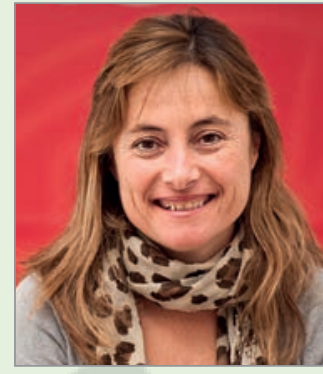

Montserrat Iracheta

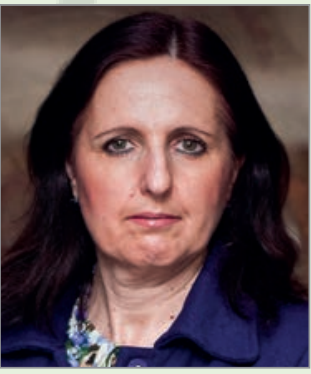

Ana Molinero
En la actualidad la hipertensión arterial (HTA) es uno de los problemas de salud pública más importantes en los países desarrollados. Se trata de una enfermedad que aumenta considerablemente el riesgo vascular y que se caracteriza por ser asintomática, fácil de detectar $\mathrm{y}$, en la mayoría de los casos, de tratar, pero que se asocia a incapacidad, disminución de la calidad de vida (puede lesionar órganos como el corazón, riñones, cerebro, retina...) y, finalmente, mortalidad prematura si no se previene y se trata de forma adecuada. Es la causa de por lo menos la mitad de las muertes por cardiopatías y por accidente cerebrovascular (1).

La prevalencia de esta patología es elevada (30-45\%) (2) tanto a nivel mundial, pues se estima que en todo el mundo hay mil millones de hipertensos, como en España, donde sería de unos 14 millones de afectados. De hecho, en nuestro país se estima que la presencia de la HTA supera el 65\% en las personas mayores de 55-60 años. A esto hay que añadir que una gran parte de los afectados por la enfermedad desconoce su existencia. Algunos estudios apuntan que solo un 65\% de las personas que padecen HTA conoce su diagnóstico y únicamente el 85\% de ellas está tratado (lo que corresponde al 55\% de la población hipertensa).

Además, a nivel poblacional el control de la enfermedad es muy bajo, puesto que de los pacientes que reciben tratamiento farmacológico, sólo se consigue el control en el 29,5\%; es decir, apenas el 16,3\% de las personas hipertensas. El estudio ENRICA (3), realizado en España entre 2008 y 2010 en cerca de 12.000 personas representativas de la población no institucionalizada de 18 y más años, también confirmó un elevado grado de desconocimiento de los pacientes sobre la existencia de su enfermedad, hasta el punto de que un $40,6 \%$ de las personas encuestadas desconocía que eran hipertenso.

Estos factores, unidos a que la HTA se puede prevenir y mejorar con cambios en el estilo de vida (dieta, ejercicio, etc.), hacen imprescindible un abordaje precoz de esta patología en el que se utilicen los recursos sanitarios de la forma más eficiente posible, especialmente en épocas como la que vivimos en la que los sistemas de salud luchan por ser sostenibles sin perder calidad en la atención a los ciudadanos. Esto implica la participación de todos aquellos agentes de salud con capacidad para aportar una acción asistencial eficaz y entre ellos es necesario tener en cuenta al farmacéutico comunitario, cuyo papel puede ser clave, no solo por la accesibilidad que ofrece la farmacia comunitaria, sino porque el propio profesional puede aportar mucho en cuanto a asesoramiento al paciente sobre medicamentos antihipertensivos y sobre la manera de evitar complicaciones a raíz de su enfermedad.

Los farmacéuticos pueden desempeñar un rol fundamental en la prevención, control y el abordaje terapéutico de la hipertensión arterial, no solo porque son los profesionales sanitarios más cercanos a los pacientes (el 90 por ciento de la población tiene una farmacia cerca de su domicilio), sino porque a ellos acuden también todos aquellos ciudadanos que no son usuarios del sistema sanitario y no están diagnosticados con una patología aunque sean susceptibles de padecerla o acumulan factores de riesgo. No en vano, más de dos millones de personas visitan diariamente las farmacias españolas, según el Consejo General de Colegios Oficiales de Farmacéuticos, y la actuación del farmacéutico comunitario puede contribuir a aumentar el diagnóstico por el médico permitiendo que personas que desconocen su condición de hipertensas o que están en riesgo puedan plantearse cambios en sus hábitos de vida para hacerlos más saludables o recibir un tratamiento al ser derivados al médico. No se debe olvidar, además, que la HTA y el riesgo vascular se asocian a otros problemas de salud como el tabaquismo, la diabetes y la dislipemia, en los que el farmacéutico comunitario puede también intervenir en colaboración con otros miembros de los equipos de salud. 
En este contexto, es importante que los farmacéuticos comunitarios seamos conscientes de la oportunidad y el reto que como profesionales de la salud nos propone la HTA. En un momento, además, en el que se fomenta el desarrollo y la prestación de servicios profesionales para reforzar el valor y el reconocimiento de nuestra profesión, esta patología nos da una oportunidad clara, por sus dimensiones e impacto, de aumentar nuestro protagonismo en beneficio de los pacientes y de la eficiencia del sistema sanitario. El cribado de pacientes con HTA, la medición y el control de la presión arterial mediante AMPA/MAPA, el cálculo del RV y la revisión del uso de los medicamentos en HTA serían cuatro de los servicios profesionales que los farmacéuticos comunitarios deberíamos prestar a las personas hipertensas y a la población en riesgo (personas con sobrepeso, con otras patologías, etc.).

Respecto al cribado, ya hemos comentado la privilegiada posición que ofrece la red de farmacias y que debería explotarse con más fuerza para mejorar la detección precoz y la educación sanitaria en la población, especialmente entre las personas jóvenes para fomentar hábitos de vida saludables que reduzcan el riesgo de un episodio vascular en edades más avanzadas. De hecho, SEFAC ya ha llevado a cabo iniciativas de este tipo como el estudio MePAFac (4) sobre factores de riesgo cardiovascular en adolescentes, del que se realizó un programa piloto en la Comunidad de Madrid en 2012 y que ahora se va a realizar a nivel nacional.

En cuanto a la medición y el control de la PA, las recomendaciones establecidas en el documento de consenso español para la evaluación y tratamiento de HTA, así como en las guías nacionales e internacionales, sugieren no basar la detección, el control y seguimiento de la HTA de forma exclusiva en la medición aislada en consulta o medición aislada en farmacia comunitaria (MAFC), sino emplear también un método complementario como la Auto Medida de la Presión Arterial (AMPA) o Medida Ambulatoria de la Presión Arterial (MAPA). Esto favorecería un mejor abordaje de la HTA, pues hay que tener en cuenta que existe una alta prevalencia del 13\% tanto de la denominada hipertensión de bata blanca (elevación de las cifras de PA en consulta frente a valores normales fuera de la misma obtenidos con AMPA o MAPA) como de la hipertensión enmascarada o hipertensión con valores normales en consulta pero elevados fuera de ella.

Esto refleja que aunque muchas veces pensemos que medir la presión arterial puede ser una tarea sencilla, no lo es y requiere una buena preparación. Es por esto que si queremos que el farmacéutico comunitario sea un agente de salud destacado en este ámbito no se debe descuidar la formación y hay que disponer de los recursos mínimos necesarios. Por este motivo, SEFAC ha puesto en marcha el programa impacHta, que se desarrollará durante 2014 y 2015 y que tiene como objetivo capacitar al farmacéutico comunitario para la prestación de los servicios de medición y control de la presión arterial mediante AMPA/MAPA y riesgo vascular. Este programa, que se lleva a cabo con la colaboración de la Sociedad Española de Hipertensión (SEH-Lelha), no solo prevé formación online, sino también dos talleres presenciales con posterior evaluación presencial y registro de al menos dos casos clínicos.

Por último, la revisión del uso de los medicamentos que hacen los pacientes hipertensos es otra labor fundamental del farmacéutico comunitario, pues como se ha expuesto en líneas precedentes, muchos de estos pacientes pueden padecer otro tipo de patologías, con el consiguiente riesgo de interacciones, reacciones adversas, etc. Es misión de nuestro colectivo, como expertos en medicamentos, garantizar un uso efectivo y seguro de los tratamientos farmacológicos.

Con todas estas acciones el farmacéutico comunitario no solo puede ayudar decisivamente a que el paciente se implique más y mejor en el conocimiento, control y seguimiento de su enfermedad, también en una mayor adherencia terapéutica. Además, la intervención farmacéutica puede ayudar a los médicos a realizar un diagnóstico más exacto de los pacientes y evaluar mejor la efectividad de la medicación y, por otra parte, el sistema sanitario en su conjunto puede ser más eficiente y optimizar recursos gracias a las mejoras producidas con la prevención, la detección precoz, el control y seguimiento y las mejoras en las tomas de los medicamentos.

En definitiva, los farmacéuticos comunitarios podemos y debemos ser un aliado necesario e, incluso, imprescindible para mejorar el abordaje de esta enfermedad y de otras muchas asociadas con ella, en colaboración con los médicos y el personal de enfermería. Citando textualmente a la presidenta de la SEH-Lehla, Nieves Martell: "Estamos ante un importante problema de salud que obliga a sumar esfuerzos para evitar riesgos asociados a las altas cifras de presión arterial y para ayudar a los pacientes, tanto para controlar su enfermedad como para que tomen conciencia de la gravedad de no hacerlo. Por eso, tenemos una excelente colaboración con los farmacéuticos puesto que los consideramos aliados absolutos”.

En nuestras manos está prepararnos para afrontar este reto con éxito.

\section{Referencias bibliográficas}

1. Victor RG, Kaplan NM. Systemic hypertension: mechanisms and diagnosis. En: Libby P, Bonow RO, Mann DL, Zipes DP, Braunwald E, [editores]. Braunwald's Heart disease. A textbook of cardiovascular medicine. Philadelphia, Pa: Saunders Elsevier; 2008. p. 1027-48.

2. Grupo de Trabajo para el manejo de la hipertensión arterial de la Sociedad Europea de Hipertensión (ESH) y la Sociedad Europea de Cardiología (ESC). Guía de práctica clínica de la ESH/ ESC para el manejo de la hipertensión arterial (2013). Hipertensión Riesg Vasc. 2013;30(Supl 3):4-91.

3. Banegas JR, Graciani A, Guallar-Castillón P, León-Muñoz LM, Gutiérrez-Fisac JL, López-García E, et al. Estudio de Nutrición y Riesgo Cardiovascular en España (ENRICA). Madrid: Universidad Autónoma de Madrid; 2011.

4. Cervero M, Molinero A, Cordero MJ, Martín A, Magro MC, Cosín A. Estudio MEPAFAC I: Medida de Presión Arterial en Adolescentes realizada por Farmacéuticos Comunitarios en centros escolares. Educación sobre factores de riesgo cardiovascular. Farmacéuticos Comunitarios 2012;4(3):108-16. 